\title{
RESPONSE OF THREE COMMON STRAWBERRY CULTIVARS TO SALT STRESS IN JORDAN
}

(Received: 20.10.2008)

\author{
By \\ S. J. Owais \\ Department of Plant Protection and Integrated Pest Management, Faculty of Agriculture, \\ Mu'tah University, Karak, Jordan
}

\begin{abstract}
Sodium chloride $(\mathrm{NaCl})$ treatments were conducted on three strawberry cultivars (Fragaria ananassa cvs. Camarosa, Albium and Ventana). The experiment was conducted in a glasshouse from the $10^{\text {th }}$ of October 2007 until the $10^{\text {th }}$ of January 2008 at Mu'tah University Agricultural Station, Karak, Jordan. Two concentrations of $\mathrm{NaCl}(30$ and $60 \mathrm{mM})$ in addition to the blank was used for 60 days. All applied treatments contained Half strength Hogland solution. Plant height, leaf weight, root length and root weight were significantly $(\mathrm{P}<0.05)$ decreased by increasing salinity level. The percentage of leaf damage was significantly $(\mathrm{P}<0.05)$ increased by increasing salinity. There were significant cultivar ax salt interaction $(\mathrm{P}<0.05)$ on root length, percentage of leaf damage and $\mathrm{N}$ accumulation in leaves and root, indicating that the cultivars responded to salt differently. Na content rose significantly in all tested cultivars, but higher concentrations were recorded in Camarosa and Ventana cultivars than in Albium. In conclusion, the results indicated that Albium cultivar is less affected by $\mathrm{NaCl}$ stress at early growth stage of plant development than Camarosa and Ventana cultivars, indicating that Albium cultivar has a genetic potential for salt tolerance, at least at this stage of its life cycle.
\end{abstract}

Key words: growth traits, mineral composition, salt stress, strawberry.

\section{INTRODUCTION}

Salinity problems are increasingly limiting to crop production at a global level, affecting about 95 million hectares worldwide (Szablocs, 1994). Many management practices have been adopted by soil scientists to overcome salinity problem, such as leaching salts from the soil by irrigation (Meri, 1984) and/or selecting more salt tolerant genotypes (Jaradat et al., 2004 and El-Hendawy et al., 2005). However, the cost and availability of irrigation water under semi-arid conditions make the irrigation highly expensive and have not given satisfactory results for a large scale. Therefore, the least expensive measure is to grow cultivars tolerant to salt stress (Shannon, 1985 and Noble and Rogers, 1992).

Salinity causes several problems for plant growth and development (Shannon et al., 1994). Strawberry is an example of a salt sensitive species, but differences between cultivars are existing (Goncharova and Dobrenkova, 1981; and Martinez Barroso and Alvarez, 1997). Investigations on tolerance to saline environments frequently point to restricted ion accumulation and organic solute synthesis as major adaptations leading to salt resistance in glycophytes (Greenway and Munns, 1980). Moreover, multiple genes that seem to increase salinity tolerance and certain proteins involved in salinity-stress protection have also been recognized (Bohnert and Jensen, 1996). Other workers have linked $\mathrm{NaCl}$ stress with macro-nutrient deficiencies, e.g. high $\mathrm{NaCl}$ concentration has been shown to induce nitrogen and calcium deficiencies, in wheat and barley (Ehret et al., 1990), maize (Evlagon et al., 1990), and in tomato (Navarro et al., 2000).

Crop performance may be adversely affected by salinity-induced nutritional disorders. These disorders may result from the effect of salinity on nutrient availability, competitive uptake, transport or partitioning within the plant (Grattan and Grieve, 1999). Therefore, many experiments have been designed to study the effects of salinity on growth parameters and mineral nutrition of commercial crops (Kaya et al., 2001). Salinity affects the crop during vegetative and reproductive stages and therefore causes reductions in both dry biomass and crop yield (Aslam et al., 1993). One important deleterious effect of elevated salinity is leaf senescence; 
young seedlings and plants at the flowering stage seem to be more sensitive than those at more mature growth stages (Lutts et al., 1995). One of the major factors inducing leaf senescence is the decrease of chlorophyll content under saline conditions (Chen et al., 1991). Leaf senescence, membrane permeability is also affected by high salt concentration (Dhindsa et al., 1981).

Screening is an essential part to identify salt tolerant genotypes in strawberry and other crops (Gaya et al., 1997; Pecetti and Gorham, 1997; Saied et al., 2005; Turhan and Eris, 2006). Field screening procedures in saline soils are confronted by high spatial and temporal variability problems (Hajrasuliha et al., 1980 and Richards, 1983). Hence, most screening experiments for salttolerant genotypes have been conducted under either in vitro or controlled environmental conditions (Kingsbury and Epstein, 1984; Munns et al., 2000; Saied et al., 2005 and Keutgen and Pawelzik, 2008).

In Jordan, salinity is one of the major soil problems that limits the crop production especially in irrigated areas. The aims of this study were to investigate the morphological and mineral composition changes in three commonly cultivated strawberry cultivars in Jordan induced by sodium chloride $(\mathrm{NaCl})$ stress and to assess their potential in tolerating salt stress at early stage of plant development.

\section{MATERIALS AND METHODS}

\subsection{Plant materials and growth conditions}

Plantlets of strawberry cultivars (Fragaria ananassa cvs. Camarosa, Albium and Ventana) were grown in 1 liter pots in glasshouse in a mixture of three materials, soil, perlite and peatmoss, with equal ratios. The experiment was conducted from the $10^{\text {th }}$ October 2007 until the $10^{\text {th }}$ January 2008 at Mu'tah University Agricultural Station, Karak, Jordan. The air temperature ranged from 23 to $27{ }^{\circ} \mathrm{C}$ during the day and 18 to $22{ }^{\circ} \mathrm{C}$ during the night. Relative humidity fluctuated between 60 and $70 \%$ at day/night. When the plantlets had developed 4-5 true leaves (30 days after planting in pots), applications of modified Hogland solution (halfstrength) containing 0 (control), 30 and $60 \mathrm{mM}$ $\mathrm{NaCl}$ were commenced. Pots were irrigated once every other day with distilled water $(0 \mathrm{mM})$, or one of the saline solutions ( 30 and $60 \mathrm{mM} \mathrm{NaCl}$ ), until the growing medium reaches $85 \%$ from its available water. Weeding pots were done frequently. A factorial experiment with two factors (cultivars and salinity) with three levels was used. The treatment combinations were replicated five times and arranged in a completely randomized design (CRD).

\subsection{Growth measurements}

At the end of the experiment (60 days after salinization), plant height $(\mathrm{cm})$ was measured and plants were separated into leaves and root parts. Thereafter, data were collected for the following parameters: leaf fresh and dry weight, root fresh dry weight and the percentage of leaf damage. Total leaf area was measured using a planimeter (Plancom KP-90N). For standardizing data, the percentage of reduction in each trait on comparison to the control was calculated using the following formula (Ghoulam et al., 2002):

Relative reduction $(\%)=[1-$ salanized/control 100\%]

2.3. Analysis of $\mathrm{Na}^{+}, \mathrm{K}^{+}, \mathrm{Ca}^{++}, \mathrm{Mg}^{++}, \mathbf{N}$ and $\mathrm{P}$ content in leaves and roots

Sodium, potassium, calcium and magnesium concentrations were estimated from samples, harvested at the end of the experiment from leaf and root parts. Collected samples were carefully rinsed with distilled deionized water, and then dried at $75{ }^{\circ} \mathrm{C}$ for $72 \mathrm{~h}$. One $\mathrm{g}$ of dried samples were digested with concentrated $\mathrm{HNO}_{3}$ and $\mathrm{HClO}_{4}$ $(4: 2 \mathrm{ml})$ at $180^{\circ} \mathrm{C}$ for $12 \mathrm{~h}$. Concentrations of $\mathrm{Na}^{+}$, $\mathrm{K}^{+}, \mathrm{Ca}^{++}$and $\mathrm{Mg}^{++}$were estimated by atomic absorption spectrometery (Perkin-Elmer Atomic Absorption Spectrophotometer, Model 5000: Perkin-Elmer; Norwalk, CT). N concentration was estimated by the standard macro-Kjeldahl procedure by digesting $1 \mathrm{~g}$ of the samples in $\mathrm{H}_{2} \mathrm{SO}_{4}$. P- content of leaf and root was determined using the method of Olsen's $\left(\mathrm{NaHCO}_{3}\right)$ using spectrophotometer. All procedures used in chemical analyses are reported in Tandon (1995).

\subsection{Statistical analysis}

Analysis of variance (ANOVA) was used to test for cultivar and salt treatment effects as well as their interaction. Data were analyzed by two way analysis of variance using the statistical package MSTAT-C, and the differences between the means were compared using least significant difference at $\mathrm{P} \leq 0.05$ (Steel and Torrie, 1980).

\section{RESULTS}

\subsection{Effect of salt treatment on growth}

Strawberry cultivars showed highly significant $(\mathrm{P}<0.01)$ differences for all growth traits investigated in this experiment, except root length. Moreover, analysis of variance indicated significant salinity effect on all tested parameters (Table 1). Effect of different salinity levels on growth parameters of the three tested cultivars is presented in (Table 2). Root length was inhibited 
severely by salinity levels more than plant height at high salt level. The low salinity treatment (30 $\mathrm{mM})$ reduced these parameters to a lesser extent than high salinity treatments $(60 \mathrm{mM})$. At 30 and $60 \mathrm{mM} \mathrm{NaCl}$, plant height was reduced by 4.21 and $12.97 \%$; leaf fresh weight reduced by 16.96 and $42.62 \%$; leaf dry weight was reduced by 16.42 and $31.59 \%$, root length was reduced by 12.02 and $17.18 \%$, root fresh weight by 18.21 and $63.13 \%$ and root dry weight was reduced by 16.86 and $47.98 \%$ as compared with the control, respectively. Leaf damage was significantly increased by increasing salt stress, the percentage of leaf damage was 4.6, 68.70 and $98.29 \%$ at 0 , 30 and $60 \mathrm{mM} \mathrm{NaCl}$, respectively. The very low percentage of leaf damage in the control may be due to the surrounding environmental factors.

There was a significant interaction between cultivar and salinity treatment on root fresh and dry weight and leaf damage. However, interactive effect was not significant on other tested parameters. The interactive effects of cultivars and salt levels on growth parameters are shown in (Table 3). Root fresh weight, root dry weight and leaf damage in all cultivars significantly decreased with increasing salinity level. However, among cultivars, the reductions in all these traits were less in Albium cultivar when it was irrigated by the highest salinity level (Table 1). At $60 \mathrm{mM}$ salinity, root fresh weight was reduced by 69.60 , 49.86 and $61.64 \%$, root dry weight reduced by 66.18, 28.87 and $36.50 \%$ in Camarosa, Albium and Ventana, respectively (Table 4).

\subsection{Salinity effects on leaf damage}

The experimental plants displayed optimum growth before the salinization commenced. For comparison of the cultivars, effects of salinity treatment on the pecentage of leaf damage are shown in (Table 1). The results of statistical analysis are also presented in Table 3 to compare the percentage of leaf damage caused by salinity in the three tested cultivars. The results showed that the percentage of leaf damage increased with increasing salinity (Table 2). High salinity treatment resulted in a complete leaf damage of the plants 60 days after salinization. For the three cultivars, the percentage of leaf damage at $30 \mathrm{mM}$ was significantly different from those in the control. The percentage of leaf damage at $30 \mathrm{mM}$ was the lowest in Albium and the highest in Camarosa and Ventana; the leaf damage was 84.20, 52.32 and $69.58 \%$ in Camarosa, Albium and Ventana, respectively. Albium was the less affected cultivar by leaf damage at intermediate salinity level $(30 \mathrm{mM} \mathrm{NaCl})$, however all leaves in the three cultivars were completely burned at high salinity treatments $(60 \mathrm{mM})$ (Tables 3 and 4$)$.

\subsection{Effect of salt stress on the leaf and root mineral content}

$\mathrm{Na}$ content rose significantly in Albium and higher concentrations were recorded in Camarosa and Ventana cultivar. The leaf and root concentrations of $\mathrm{K}, \mathrm{Ca}, \mathrm{Mg}$ and $\mathrm{P}$ were decreased in all tested cultivars, although non-significant. Accumulation of $\mathrm{N}$ and $\mathrm{P}$ was significantly reduced by increasing salt stress with considerable variation among varieties for $\mathrm{N}$ content. The lowest reductions in $\mathrm{N}$ values were found in Albium cultivar and the highest reductions were detected in Camarosa and Ventana.

\section{DISCUSSION}

The analysis of variance revealed high significant differences among the three cultivars for the studied parameters (plant height, leaves fresh and dry weight, root fresh and dry weight and percentage of leaves damage) and leaves and root mineral composition. This indicated that an adequate amount of variability was present in the tested strawberry cultivars. The genetic variation was also reflected in the differences observed among the cultivars for salt tolerance. Similarly, a wide genotypic variation for salt tolerance in strawberry cultivars was observed in previous studies (Goncharova and Dobrenkova, 1981; Martinez and Alvarez, 1997).

In the current study, only one salt $(\mathrm{NaCl})$ rather than a mixture of salts $\left(\mathrm{NaCl}, \mathrm{CaSO}_{4}\right.$, $\mathrm{MgCl}_{2}, \mathrm{Na}_{2} \mathrm{SO}_{4}$ ) was used to impose salt stress due to high $\mathrm{Na}^{+}$and $\mathrm{Cl}^{-}$ion toxicity effects on the plant tissue. Similarly, most researchers (Kaya et al., 2002 ( $\mathrm{a}$ and b); Turhan and Eris, 2006; Tuna et al., 2007; Keutgen and Pawelzik, 2008; ) used $\mathrm{NaCl}$ as a single salt in salt stress experiments due to high toxicity effect of chloride and sodium. Since saline field soils contain a mixture of salts rather than a single salt, it seems that plants under field conditions are more salt tolerant in comparison with $\mathrm{NaCl}$ salinity. It may be related to the presence of $\mathrm{Ca}, \mathrm{Mg}$ and $\mathrm{SO} 4$ ions that reduce the $\mathrm{Na}^{-}$and $\mathrm{Cl}^{-}$deleterious effects on plants (Volkmar et al., 1998).

All studied growth parameters measured were adversely affected by increasing salt level. In saline environment where salts are present in higher concentrations, the mechanisms by which 
Response of three common strawberry cultivars ...............................................................

Table (1): Analysis of variance for the effects of cultivar and salt level on some leaf and root characteristics

\begin{tabular}{|c|c|c|c|c|}
\hline Trait & Cultivar (2 d.f.) & Salt level (2 d.f.) & Cultivar $\times$ salt level (4 d.f.) & Error (36 d.f.) \\
\hline Plant height (cm) & $20.478 *$ & $18.344 *$ & 3.222 & 87.100 \\
\hline Leaves fresh weight (g) & 73.160* & $102.325 * *$ & 47.590 & 309.003 \\
\hline Leaves dry weight (g) & $26.606 * *$ & $12.051 * *$ & 6.307 & 41.134 \\
\hline Root length $(\mathrm{cm})$ & 142.178 & 172.044* & 87.422 & 29.600 \\
\hline Root fresh weight (g) & 681.080** & $787.937 * *$ & 234.571* & 806.925 \\
\hline Root dry weigh (g) & $35.781 * *$ & $31.483 * *$ & 13.511* & 39.595 \\
\hline$\%$ of leaves damage & 1126.897* & $68804.561 * *$ & 11468.369* & 4347.532 \\
\hline
\end{tabular}

*,** Significant at 0.05 and 0.01 probability level, respectively

Table (2): Analysis of variance for the effects of cultivar and salt level on leaf and root mineral composition.

\begin{tabular}{|c|c|c|c|c|c|}
\hline Plant part & Mineral & Cultivar (2 d.f.) & Salt level (2 d.f.) & Cultivar $\times$ salt level $(4$ d.f. $)$ & Error (18 d.f.) \\
\hline \multirow[t]{6}{*}{ Leaves } & $\mathbf{N a}$ & $3.318 * *$ & $16.041 * *$ & $3.736 * *$ & 2.454 \\
\hline & $\mathbf{K}$ & $2.167 * *$ & 0.250 & 0.103 & 1.064 \\
\hline & $\mathbf{C a}$ & $1.812 * *$ & 0.175 & 0.037 & 0.777 \\
\hline & Mg & 0.009 & 0.156 & 0.005 & 0.629 \\
\hline & $\mathbf{N}$ & $13.014 * *$ & $22.477 * *$ & $14.356 * *$ & 1.349 \\
\hline & $\mathbf{P}$ & $0.008 *$ & 0.000 & 0.000 & 0.018 \\
\hline \multirow[t]{6}{*}{ Root } & $\mathbf{N a}$ & 2.276* & $10.357 * *$ & $4.227 * *$ & 4.123 \\
\hline & $\mathbf{K}$ & $4.553 * *$ & 0.064 & 0.004 & 0.864 \\
\hline & $\mathbf{C a}$ & $0.782 * *$ & 0.064 & 0.011 & 0.346 \\
\hline & Mg & 0.023 & 0.093 & 0.052 & 1.127 \\
\hline & $\mathbf{N}$ & $1.286 * *$ & $0.339 * *$ & $0.148 *$ & 0.184 \\
\hline & $\mathbf{P}$ & $0.067 * *$ & $0.015 * *$ & 0.001 & 0.022 \\
\hline
\end{tabular}




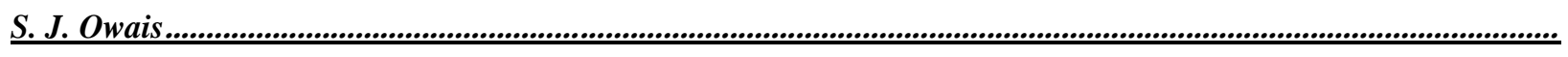

Table ( 3): Effect of different salinity levels on leaf and root parameters of the three strawberry cultivars.

\begin{tabular}{|c|c|c|c|c|c|c|c|c|c|}
\hline Cultivars & $\begin{array}{l}\text { Plant height } \\
\text { (cm) }\end{array}$ & $\begin{array}{l}\text { Leaves fresh } \\
\text { weight (g) }\end{array}$ & $\begin{array}{l}\text { Leaves dry } \\
\text { weight (g) }\end{array}$ & $\begin{array}{l}\text { Root } \\
\text { length (cm) }\end{array}$ & $\begin{array}{l}\text { Root fresh } \\
\text { weight (g) }\end{array}$ & $\begin{array}{l}\text { Root dry } \\
\text { weight (g) }\end{array}$ & $\begin{array}{l}\text { Area of fired } \\
\text { leaves }\left(\mathrm{cm}^{2}\right)\end{array}$ & $\begin{array}{l}\text { Total leaf area } \\
\left(\mathrm{cm}^{2}\right)\end{array}$ & $\begin{array}{lll}\begin{array}{l}\% \\
\text { of }\end{array} \\
\text { damage }\end{array}$ \\
\hline Camarosa & $12.00 a$ & $3.17^{b}$ & $2.05 \mathrm{~b}$ & $22.20 \mathrm{a}$ & $14.94 \mathrm{a}$ & $4.00 \mathrm{a}$ & 174.54 & 110.90 & 63.54a \\
\hline Albium & $11.93 a$ & $7.59^{\mathrm{a}}$ & 3.85a & $24.73 a$ & $6.05 \mathrm{~b}$ & $2.04 \mathrm{~b}$ & 119.33 & 61.23 & $51.31 \mathrm{~b}$ \\
\hline Ventana & $10.30 \mathrm{~b}$ & $5.11^{\mathrm{b}}$ & $2.29 \mathrm{~b}$ & $26.53 a$ & $13.47 \mathrm{a}$ & 3.85a & 146.26 & 83.00 & 56.75ab \\
\hline $\operatorname{LSD}(0.05)$ & 1.15 & 2.17 & 0.79 & NS & 3.51 & 0.78 & - & - & 8.14 \\
\hline \multicolumn{10}{|l|}{$\begin{array}{l}\begin{array}{l}\text { Salt level } \\
\text { (mM) }\end{array} \\
\end{array}$} \\
\hline$\underline{0}$ & $11.87 \mathbf{a}$ & $8.61^{\mathrm{a}}$ & 4.02 & $27.13 \mathrm{a}$ & $15.76 \mathrm{a}$ & $4.21 \mathrm{a}$ & 210.39 & $9 . .69$ & $4.607 \mathrm{c}$ \\
\hline \multirow[t]{2}{*}{30} & 11.37ab & $7.15^{\mathrm{a}}$ & 3.36 & $23.87 \mathrm{a}$ & $12.89 \mathrm{a}$ & $3.50 \mathrm{a}$ & 177.58 & 122.0 & $68.70 \mathrm{~b}$ \\
\hline & (4.21) & $(16.96)$ & (16.42) & $(12.02)$ & (18.21) & $(16.86)$ & & & $(-1391.21)$ \\
\hline \multirow[t]{2}{*}{60} & $10.33 b$ & $4.94^{\mathrm{b}}$ & 2.75 & $22.47 \mathrm{a}$ & $5.81 \mathrm{~b}$ & $2.19 \mathrm{~b}$ & 125.65 & 123.5 & $98.29 \mathrm{a}$ \\
\hline & $(\mathbf{1 2 . 9 7 )}$ & $(\mathbf{4 2 . 6 2 )}$ & $(\mathbf{3 1 . 5 9 )}$ & (17.18) & $(63.13)$ & (47.98) & & & $(-2033.79)$ \\
\hline $\mathrm{LSD} \leq 0.05$ & 1.15 & 2.17 & 0.79 & $3.96 \mathrm{a}$ & 3.51 & 0.78 & - & - & 8.14 \\
\hline Interaction & NS & NS & NS & NS & * & * & - & - & $*$ \\
\hline
\end{tabular}

Values in the brackets indicate \% reduction over their respective controls

$*, * *$ Significant at $5 \%$ and $1 \%$ probability levels. respectively; NS= non-significant at $\mathrm{P}<0.05$

Means followed by the same letter within each column are not significantly different at $\mathrm{P}<0.05$ according to LSD.

Table (4): Interactive effects of cultivars. and salt levels on leaf, root characteristics and leaf damage of the three studied cultivars.

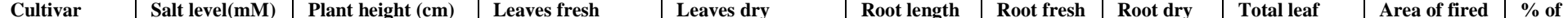

\begin{tabular}{|c|c|c|c|c|c|c|c|c|c|c|}
\hline \multirow{3}{*}{ Camarosa } & 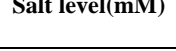 & 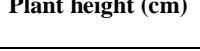 & weight (g) & weight $(\mathrm{g})$ & $(\mathbf{c m})$ & weight (g) & weigh (g) & area $\left(\mathrm{cm}^{2}\right)$ & leaves $\left(\mathrm{cm}^{2}\right)$ & leaf firing \\
\hline & 30 & $\begin{array}{l}12.60^{\mathrm{a}} \\
(-1.61)\end{array}$ & $\begin{array}{c}8.31^{\mathrm{a}} \\
(22.55) \\
\end{array}$ & $\begin{array}{l}4.33^{\mathrm{a}} \\
(\mathbf{4 . 6 3})\end{array}$ & $\begin{array}{l}21.80 \mathrm{a} \\
(6.84)\end{array}$ & $\begin{array}{l}18.86^{\mathrm{a}} \\
(5.23)\end{array}$ & $\begin{array}{l}\begin{array}{l}\text { 4.67ab } \\
(15.09)\end{array} \\
\end{array}$ & 218.76 & 184.20 & $\begin{array}{c}84.20 b \\
(-1211.53)\end{array}$ \\
\hline & 60 & $\begin{array}{l}10.80^{\mathrm{a}} \\
(12.90)\end{array}$ & $\begin{array}{c}3.71^{\mathrm{a}} \\
(65.42)\end{array}$ & $\begin{array}{l}2.68^{\mathrm{a}} \\
(40.97)\end{array}$ & $\begin{array}{l}21.40 \mathrm{a} \\
(8.55)\end{array}$ & $\begin{array}{c}6.05^{\mathrm{c}} \\
(69.60)\end{array}$ & $\begin{array}{l}1.86 d \\
\text { (66.18) }\end{array}$ & 132.90 & 132.90 & $\begin{array}{l}100.00 \mathrm{a} \\
(-1457.63)\end{array}$ \\
\hline \multirow{2}{*}{ Albiumm } & 30 & $\begin{array}{l}10.30^{\mathrm{a}} \\
(8.04)\end{array}$ & $\begin{array}{r}4.69^{\mathrm{a}} \\
(24.84) \\
\end{array}$ & $\begin{array}{l}2.23^{\mathrm{a}} \\
(11.51)\end{array}$ & $\begin{array}{l}24.40 \mathrm{a} \\
(6.87) \\
\end{array}$ & $\begin{array}{l}7.10^{\mathrm{bc}} \\
(3.53) \\
\end{array}$ & $\begin{array}{l}2.03 d \\
(15.06)\end{array}$ & 114.66 & 59.99 & $\begin{array}{c}52.32 d \\
(-1157.69)\end{array}$ \\
\hline & 60 & $\begin{array}{r}9.40^{\mathrm{a}} \\
(16.07) \\
\end{array}$ & $\begin{array}{r}4.40^{\mathrm{a}} \\
(29.49) \\
\end{array}$ & $\begin{array}{l}2.11^{\mathrm{a}} \\
(16.27) \\
\end{array}$ & $\begin{array}{l}23.60 a \\
(9.92) \\
\end{array}$ & $\begin{array}{c}3.69^{\mathrm{c}} \\
(49.86) \\
\end{array}$ & $\begin{array}{l}1.70 d \\
(28.87)\end{array}$ & 121.82 & 118.7 & $\begin{array}{c}\text { 97.44ab } \\
(-2242.31)\end{array}$ \\
\hline \multirow{2}{*}{ Ventana } & 30 & $\begin{array}{l}11.20^{\mathrm{a}} \\
(6.97)\end{array}$ & $\begin{array}{r}8.43^{\mathrm{a}} \\
(4.75) \\
\end{array}$ & $\begin{array}{l}3.52^{\mathrm{a}} \\
(29.46) \\
\end{array}$ & $\begin{array}{l}\text { 25.40a } \\
\text { (20.13) } \\
\end{array}$ & $\begin{array}{l}12.71^{\mathrm{b}} \\
(36.51)\end{array}$ & $\begin{array}{l}3.81 b c \\
(19.62)\end{array}$ & 175.05 & 121.8 & $\begin{array}{c}69.58 c \\
(-2047.53) \\
\end{array}$ \\
\hline & 60 & $\begin{array}{l}10.80^{\mathrm{a}} \\
(10.00)\end{array}$ & $\begin{array}{r}6.71^{\mathrm{a}} \\
(24.18) \\
\end{array}$ & $\begin{array}{l}3.45^{\mathrm{a}} \\
(30.86) \\
\end{array}$ & $\begin{array}{l}22.40 a \\
(29.56) \\
\end{array}$ & $\begin{array}{r}7.68^{\mathrm{bc}} \\
(61.64) \\
\end{array}$ & $\begin{array}{l}\text { 3.01cd } \\
(36.50) \\
\end{array}$ & 121.82 & 118.7 & $\begin{array}{r}97.44 a b \\
(-2907.41) \\
\end{array}$ \\
\hline LSD $\leq 0.05$ & & NS & NS & NS & NS & $*$ & * & - & - & * \\
\hline
\end{tabular}

Significant at $5 \%$ and $1 \%$ probability levels. respectively; $\mathrm{NS}=$ non-significant at $\mathrm{P}<0.05$

Means followed by the same letter within each column are not significantly different at $\mathrm{P}<0.05$ according to LSD. 
Table (5): Effect of different salinity levels on leaf mineral composition of the three strawberry cultivars.

\begin{tabular}{|c|c|c|c|c|c|c|}
\hline \multicolumn{7}{|c|}{ Mineral concentration g/100g dry weight basis } \\
\hline Cultivars & $\mathrm{Na}+$ & $\mathbf{K}+$ & $\mathrm{Ca}++$ & Mg++ & $\mathbf{N}$ & $\mathbf{P}$ \\
\hline Camarosa & 1.601a & $0.830 b$ & $1.013 \mathrm{c}$ & 0.938 & $1.927 b$ & 0.1705 \\
\hline Albium & $0.743 c$ & $1.465 \mathrm{a}$ & $1.647 a$ & 0.980 & $1.017 \mathrm{c}$ & 0.1561 \\
\hline Ventana & 1.179b & 1.389a & $1.336 \mathrm{~b}$ & 0.980 & $2.716 a$ & 0.1705 \\
\hline LSD (0.05) & 0.365 & 0.241 & 2.054 & NS & 0.2712 & NS \\
\hline \multicolumn{7}{|c|}{ Salt level (mM) } \\
\hline $\mathbf{0}$ & $0.274 \mathrm{c}$ & 1.316a & $1.444 a$ & 1.063 & 3.152a & 0.1464 \\
\hline 30 & $1.093 b$ & $1.274 \mathrm{a}$ & $1.293 \mathrm{a}$ & 0.951 & $1.473 \mathrm{~b}$ & 0.1544 \\
\hline 60 & $2.156 a$ & $1.094 a$ & $1.258 \mathrm{a}$ & 0.879 & $1.035 c$ & 0.1557 \\
\hline LSD $\leq 0.05$ & 0.365 & NS & NS & NS & 0.271 & NS \\
\hline Interaction & & & & & & \\
\hline
\end{tabular}

*, ** Significant at $5 \%$ and $1 \%$ probability levels. respectively; $\mathrm{NS}=$ non-significant at $\mathrm{P}<0.05$

Table (6): Interactive effects of cultivar and salt levels on leaf mineral composition of the three studied cultivars.

\begin{tabular}{|c|c|c|c|c|c|c|c|}
\hline \multicolumn{8}{|c|}{ Mineral concentration g/100g dry weight basis } \\
\hline Cultivar & $\begin{array}{l}\text { Salt Level } \\
\text { Concntration } \\
\text { (UM) }\end{array}$ & $\mathrm{Na}^{+}$ & $\mathbf{K}^{+}$ & $\mathrm{Ca}^{++}$ & Mg++ & $\mathbf{N}$ & $\mathbf{P}$ \\
\hline \multirow[t]{3}{*}{ Camarosa } & $\mathbf{0}$ & 0.318d & 0.856 & 1.133 & 1.050 & $2.918 b$ & $\mathbf{0 . 1 3 0 0}$ \\
\hline & 30 & $1.336 \mathrm{c}$ & 0.858 & 0.989 & 0.906 & $1.898 \mathrm{c}$ & 0.1327 \\
\hline & 60 & 3.150a & 0.775 & 0.945 & 0.857 & $0.9640 \mathrm{e}$ & 0.1270 \\
\hline \multirow[t]{3}{*}{ Albiun } & $\mathbf{0}$ & 0.390d & 1.649 & 1.814 & 1.084 & 1.204de & 0.1492 \\
\hline & 30 & $0.743 \mathrm{~cd}$ & 1.526 & 1.606 & 0.959 & $1.003 \mathrm{e}$ & 0.1550 \\
\hline & 60 & $1.096 \mathrm{c}$ & 1.220 & 1.522 & 0.896 & $0.8437 \mathrm{e}$ & 0.1641 \\
\hline \multirow[t]{3}{*}{ Ventana } & $\mathbf{0}$ & 0.113d & 1.444 & 1.385 & 1.056 & 5.333a & 0.1601 \\
\hline & 30 & $1.201 \mathrm{c}$ & 1.437 & 1.315 & 0.987 & $1.517 \mathrm{ed}$ & 0.1756 \\
\hline & 60 & $2.223 b$ & 1.287 & 1.308 & 0.882 & $1.297 \mathrm{de}$ & 0.1759 \\
\hline LSD (0.05) & & 0.633 & NS & NS & NS & 0.4698 & NS \\
\hline
\end{tabular}

*, ** Significant at $5 \%$ and $1 \%$ probability levels. respectively; $N S=$ non-significant at $\mathrm{P}<0.05$

Means followed by the same letter within each column are not significantly different at $\mathrm{P}<0.05$ according to LSD. 
Table (7): Effect of different salinity levels on root mineral composition of the three strawberry cultivars.

\begin{tabular}{|c|c|c|c|c|c|c|}
\hline \multirow[b]{2}{*}{ Cultivars } & \multicolumn{6}{|c|}{ Mineral concentration $\mathrm{g} / \mathbf{1 0 0 g}$ dry weight basis } \\
\hline & $\mathrm{Na}^{+}$ & $\mathbf{K}^{+}$ & $\mathrm{Ca}^{++}$ & $\mathbf{M g}^{++}$ & $\mathbf{N}$ & $\mathbf{P}$ \\
\hline Camarosa & $1.414 a$ & 1.446a & $1.417 a$ & $0.996 a$ & $1.189 b$ & $0.1345 b$ \\
\hline Albium & $0.899 b$ & $0.616 b$ & $1.053 b$ & $1.067 a$ & $1.464 a$ & $0.2411 \mathrm{a}$ \\
\hline Ventana & 0.732b & $0.540 b$ & $1.059 b$ & $1.029 a$ & $0.930 c$ & $0.1361 b$ \\
\hline LSD(0.05) & 0.474 & 0.217 & 1.417 & NS & 0.099 & $\mathbf{0 . 0 3 1 3 2}$ \\
\hline \multicolumn{7}{|l|}{ Salt level (mM) } \\
\hline $\mathbf{0}$ & $0.225 \mathrm{c}$ & $0.922 a$ & $1.236 a$ & $1.09 a$ & $1.331 \mathrm{a}$ & 0.1945a \\
\hline 30 & $1.081 b$ & $0.876 a$ & $1.176 a$ & $1.05 a$ & $1.196 b$ & $0.1789 a$ \\
\hline 60 & $1.738 \mathrm{a}$ & $0.804 a$ & $1.117 a$ & $0.951 \mathrm{a}$ & $1.056 c$ & 0.1383b \\
\hline LSD (0.05) & 0.474 & NS & NS & NS & 0.099 & 0.03132 \\
\hline Interaction & $* *$ & NS & NS & NS & $*$ & $* *$ \\
\hline
\end{tabular}

$*, * *$ Significant at $5 \%$ and $1 \%$ probability levels. respectively; $\mathrm{NS}=$ non-significant at $\mathrm{P}<0.05$

Means followed by the same letter within each column are not significantly different at $\mathrm{P}<0.05$ according to LSD.

Table (8): Interactive effects of cultivars and salt levels on root mineral composition of the three studied cultivars.

\begin{tabular}{|c|c|c|c|c|c|c|c|}
\hline \multirow[b]{2}{*}{ Cultivar } & \multirow[b]{2}{*}{ Salt level } & \multicolumn{6}{|c|}{ Mineral concentration $\mathrm{g} / 100 \mathrm{~g}$ dry weight basis } \\
\hline & & $\mathrm{Na}^{+}$ & $\mathbf{K}^{+}$ & $\mathrm{Ca}^{++}$ & $\mathbf{M g}^{++}$ & $\mathbf{N}$ & $\mathbf{P}$ \\
\hline \multirow[t]{3}{*}{ Camarosa } & $\mathbf{0}$ & $0.060 d$ & 1.492 & 1.461 & 1.017 & $1.45 a$ & $0.1513 a$ \\
\hline & 30 & $1.291 b$ & 1.444 & 1.420 & 0.985 & $1.186 b$ & $0.1456 a$ \\
\hline & 60 & 2.891a & 1.404 & 1.369 & 0.986 & $0.932 \mathrm{~cd}$ & $0.1065 a$ \\
\hline \multirow[t]{3}{*}{ Albium } & $\mathbf{0}$ & $0.463 \mathrm{~cd}$ & 0.667 & 1.133 & 1.118 & $1.505 a$ & $0.2629 a$ \\
\hline & 30 & $0.976 b c$ & 0.639 & 1.019 & 1.086 & $1.467 a$ & $0.22508 \mathrm{a}$ \\
\hline & 60 & $1.257 \mathrm{bc}$ & 0.543 & 1.007 & 0.999 & $1.421 \mathrm{a}$ & $0.2096 a$ \\
\hline \multirow[t]{3}{*}{ Ventana } & $\mathbf{0}$ & 0.153d & 0.608 & 1.114 & 1.133 & $1.037 b c$ & $0.1694 a$ \\
\hline & 30 & 0.976bc & 0.545 & 1.088 & 1.086 & $0.936 \mathrm{~cd}$ & $0.1402 a$ \\
\hline & 60 & $1.066 b c$ & 0.465 & 0.975 & 0.868 & 0.816d & $0.09877 a$ \\
\hline LSD (0.05) & & 0.821 & NS & NS & NS & 0.172 & NS \\
\hline
\end{tabular}

salinity affects plant growth can be probably attributed to osmotic, specific ion and nutritional imbalance effects; properly all occurring simultaneously (Flowers and hajibagheri, 2001). The reduction in growth was explained by reducing osmotic potential in the soil, which leads to a decrease in water uptake by the root, reduced transpiration and closure of stomata, which is associated with the reduced growth (Levitt, 1980). The growth reduction is also induced by a suppression of nutrient absorption due to uptake of $\mathrm{Na}^{+}$and $\mathrm{Cl}^{-}$in competition with nutrient ions (Levitt, 1980 and Salisbury and Ross, 1992).The reduction of the accumulation of $\mathrm{N}$ and $\mathrm{P}$ in leaves 
and roots of the three strawberry cultivars presumably explained the reduction in growth parameters. Moreover, according to Kaya et al. (2002 a and b) and Saied et al., (2005), salt treatments increase $\mathrm{Na}^{+}$and $\mathrm{Cl}^{-}$accumulation and toxic effects related to the accumulation of these ions. Salt tolerant genotypes could adjust to salt stress by lowering tissue osmotic potential with the accumulation of inorganic ions (such as $\mathrm{Na}^{+}$, $\mathrm{K}^{+}$and $\mathrm{Cl}^{-}$) as well as organic solutes such as proline, glycinebetaine, sucrose and other sugarrelated compounds in root Husaini and Abdin 2008 and (Chen and Murata2008; Jamalian et al ., 2008).

Leaf and root weight were used to evaluate cultivars for salt tolerance. Generally, the values of the two parameters decreased with increasing salinity level. However, root weight showed greater reduction than leaf weight. In earlier studies, the root of seedlings was found to be more sensitive than the leaves in stawberry (Keutgen and Pawelzik, 2008) and vegetable crops (Shannon and Grieve, 1999).

On the basis of the reduction of root weight, Albium cultivar could be declared as relatively tolerant, while Camarosa and Ventana cultivars as sensitive ones. Beside reducing growth, raised salinity had significantly increased leaf damage. At high salinity level, almost all leaves of salt affected plants died or developed very sever leaf burn symptoms. Leaf burning is one of the harmful effects of increased salinity due to decreasing chlorophyll content and increasing membrane permeability (Chen et al., 1991; Kaya et al., 2001; and Munns, 2002). In many crops salinity tolerance may depend on the efficiency of the root system, which can limit access of $\mathrm{Na}$ to the aerial part of the plant (Ondrasek et al., 2006, and Munns and Tester 2008). The sodium content of the salt-tolerant cultivar, Albium, was lower than those of Camarosa and Ventanna, this result may clearly show that the high growth reduction in Camarosa and Ventana may be due to excessive $\mathrm{Na}$ accumulation in leaves and roots. Salt treatment increased the absorption of $\mathrm{Na}$ at the expense of $\mathrm{K}, \mathrm{Ca}, \mathrm{Mg}, \mathrm{N}$ and $\mathrm{P}$ causing more ionic disequilibrium in Camarosa and Ventana than in Albium. Under saline conditions, the effect of ions on the absorption of other ions is also of particular interest. Ions at high concentrations in the external solution (e.g. $\mathrm{Na}^{+}$) are taken up at higher rate, which may lead to excessive accumulation in tissues. $\mathrm{Na}^{+}$my inhibit the uptake of other ions into the root and their transportation to the leaves (Kaya et al. 2002 b).

\section{Conclusion}

Growth parameters such as fresh and dry weight of leaves and roots were inhibited severely by increasing $\mathrm{NaCl}$ level. The low $\mathrm{NaCl}$ treatment $(30 \mathrm{mM})$ reduced these parameters to a lesser extent than high salinity treatments $(60 \mathrm{mM})$. Moreover, high $\mathrm{NaCl}$ up to $60 \mathrm{mM}$ caused complete leaf damage on all studied cultivars, however, Albium was less affected by $30 \mathrm{mM}$ $\mathrm{NaCl}$ than Camarosa and Ventana. While salinity inhibited growth in all cultivars, Albium was found to be significantly more tolerant and less affected to $\mathrm{NaCl}$ stress than Camarosa and Ventana. The Na content and the reductions in $\mathrm{N}$ of the salt-tolerant cultivar, Albium, was lower than those of Camarosa and Ventana, which could be as an other evidence of the genetic potential of Albium cultivar for salt tolerance

\section{Acknowledgment}

The author would like to thank the Deanship of Academic Research at Mu'tah University for supporting this research (Project No. SR/120/14/328 on 13 August 2008)

\section{REFERENCES}

Aslam M., Qureshi R.H. and Ahmad N. (1993). A rapid screening technique for salt tolerance in rice (Oryza sativa L.). Plant Soil. 150: 99-107.

Bohnert H.J. and Jensen R.G. (1996). Metabolic engineering for increased salt tolerance. Aust. J. Plant Physiol. 23: 661-667.

Chen C.T., Li C.C. and Kao C.H. (1991). Senescence of rice leaves: Changes of chlorophyll, protein and polyamine contents and ethylene production during senescence of a chlorophyll-deficient mutant. J. Plant Growth Reg. 10: 201-205.

Chen T.H.H. and Murata N. (2008). Glycinebetaine: an effective protectant against abiotic stress in plants. Trends Plant Sci. 13: 499-505

Dhindsa R.S., Plumb-Dhindsa P. and Thorpe T.A. (1981). Leaf senescence correlated with increased levels of membrane permeability and lipid peroxidation, and decreased levels of superoxide dismutase and catalase. J. Exp. Bot. 32: 93-101.

Ehret D.L., Remann R.E., Harvey B.L. and Cipywnyk A. (1990). Salinity-induced calcium deficiencies in wheat and barley. Plant Soil. 128: 143-151.

El-Hendawy S.E., Hu Z., Zakout G. M., Awad A. M., Hafiz S. E. and Schmidhalter U.( 2005). 
Evaluating salt tolerance of wheat genotypes using multiple parameters. Eur. J. Agron. 22: 243-253.

Evlagon D., Ravina Y., and Neumann P.M. (1990). Interactive effects of salinity and calcium on hydraulic conductivity, osmotic adjustment and growth in primary roots of maize seedlings. Isr. J. Bot. 39: 239-247.

Flowers T.J. and Hajibagheri M.A. (2001). Salinity tolerance in Hordeum vulgare: Ion concentrations in root cells of cultivars differing in salt tolerance. Plant Soil. 231:1-9.

Gaya A.S., Schittenhelm M S. and Friedt W. (1997). Assessment of tolerance to salt stress in Kenyan tomato germplasm. Euphytica. 95: 57-66.

Ghoulam C., Foursy A. and Fares K. (2002). Effects of salt stress on growth, inorganic ions and proline accumulation in relation to osmotic adjustment in five sugar beet cultivars. Environ. Exp. Bot. 47: 39-50.

Goncharova E.A. and Dobrenkova L.G. (1981). Growth processes and yield in strawberry under conditions of drought and salinity. Trudy po Prikladnoi Botanike, Genetike i Selektsii. 70: 97-102.

Grattan S.R. and Grieve C.M. (1999). Salinity mineral nutrient relations in horticultural crops. Sci. Hort. 78: 127-157.

Greenway H., and Munns R. (1980). Mechanisms of salt tolerance in nonhalophytes. Annu. Rev. Plant Physiol. 31: 149-190.

Hajrasuliha S.N., Baniabbassi J. and Nilson D.R., (1980) . Special variability in soil sampling for salinity studies in Southwest Iran. Irrig. Sci. 1: 197-208.

Husaini A. M. and Abdin M.Z. (2008). Development of transgenic strawberry (Fragaria ananassa Duch.) plants tolerant to salt stress. Plant Sci. 174: 446-455.

Jamalian S., Tafazoli E., Eshghi S. and Davarynejad G.H. (2008). The Effect of Paclobutrazol on fruit yield, leaf mineral elements and proline content of strawberry cv. Selva under saline condition. AmericanEurasian J. Agric. Environ. Sci. 3: 118-122.

Jaradat A.A., Shahid M. and Al-Maskri A. (2004). Genetic diversity in the Batini barley landrace from Oman: II Response to salinity stress. Crop Sci. 44: 997-1007.

Kaya C., Ak B.E., Higgs D., and Murillo-Amador B. (2002 b). Influence of foliar applied calcium nitrate on strawberry plants grown under salt stress conditions. Aust. J. Exp. Agric. 42: 631-636.
Kaya C., Kirnak H. and Higgs D. (2001). Enhancement of growth and normal growth parameters by foliar application of potassium and phosphorus in tomato cultivars grown at high $(\mathrm{NaCl})$ salinity. $\mathrm{J}$. Plant Nutr. 24: 357-367.

Kaya C., Kirnak H., Higgs D. and Saltali K. (2002 a). Supplementary calcium enhances plant growth and fruit yield in strawberry cultivars grown at high $(\mathrm{NaCl})$ salinity. Sci. Hort. 93: 65-74.

Keutgen A. and Pawelzik E. (2008). Modifications of taste-relevant compounds in strawberry fruit under $\mathrm{NaCl}$ salinity. Food Chem. 105: 1487-1494

Kingsbury R.W., and Epstein E., (1984). selection for salt resistant in spring wheat. Crop Sci. 24: $310-315$.

Levitt J. (1980). Responses of Plants to Environmental Stresses. Volume II. $2^{\text {nd }}$ ed. Academic Press, New York.

Lutts S., Kinet J. and Bouharmont J. M. (1995). Changes in plant response to $\mathrm{NaCl}$ during development of rice varieties differing in salinity resistance. J. Exp. Bot., 46: 18431852.

Martinez Barroso M.C. and Alvarez C.E., (1997). Toxicity symptoms and tolerance of strawberry to salinity in the irrigation water. Sci. Hort. 71: 177-188.

Meri A. (1984). Plant Response to Salinity: Experimental Methodology and Application to the Field Soil Salinity Under Irrigation Processes and Management. Springer Verlag, Berlin.

Munns R. (2002). Comparative physiology of salt and water stress. Plant cell Environ. 25: 239-250.

Munns R. and Tester M. (2008). Mechanisms of salinity tolerance. Annual Review of Plant Biology 59: 651-681.

Munns R., Hare R.A., James R.A. and Rebetzke G.J., (2000). Genetic variation for improving the salt tolerance of durum wheat. Aust. J. Agric. Res. 51: 69-74.

Navarro J.M., Martinez V. and Carvajal M. (2000). Ammonium, bicarbonate and calcium effects on tomato plants grown under saline conditions. Plant Sci. 157: 8996.

Noble C.L. and Rogers M.E. (1992). Arguments for the use of physiological criteria for improving the salt tolerance in crops. Plant Soil. 146: 99-107. 
Ondrasek G., Romic D., Romic M., Duralija B., and Mustac I. (2006). Strawberry Growth and Fruit Yield in a Saline Environment. Agriculturae Conspectus Scientificus.71: 155-158.

Pecetti L., and Gorham J., (1997). Screening of durum wheat germplasm for $\mathrm{Na}$ uptake under moderate salinity. Cereal Res. Commun. 25: 923-930.

Richards R.A. (1983). Should selection for yield in saline conditions be made on saline or non saline soils. Euphytica. 32: 431-438.

Saied A.S., Keutgen A.J. and Noga G. (2005). The influence of $\mathrm{NaCl}$ salinity on growth, yield and fruit quality of strawberry cvs. Elsanta and Korona. Sci. Hortic. 103: 289-303.

Salisbury F.B. and Ross C.W. (1992). Plant Physiology. $4^{\text {th }}$ ed. Wadsworth, Belmont, California

Shannon M.C. (1985). Principles and strategies in breeding for higher salt tolerance. Plant Soil. 89: 227-241.

Shannon M.C. and Grieve C.M. (1999). Tolerance of vegetable crops to salinity. Sci. Hortic. 78: 5-38.

Shannon M.C., Grieve C.M. and Francois L.E. (1994). Whole-plant response to salinity. In: Wilkinson, R.E. (Ed.), Plant $\times$ Environment
Interactions. Marcel Dekker, New York, 199-244.

Steel R.G.D. and Torrie J.H. (1980). Principle and Procedures of Statistics. Published by McGraw-hill Book Company, INC New York.

Szabolcs I. (1994). Szabolcs, Soils and salinization. In: M. Pessarakli, Editor, Handbook of Plant Crop Stress, University of Arizona, Tucson, AZ, p. 3-21.

Tandon, H. (1995). Methods for Analysis of Soil, Plants, Waters and Fertilizer. Fertilizer Development and Consultation Organization, New Delhi, India, 144. pp

Tuna A.L., Kaya C., Ashraf M., Altunlu H., Yokas I. and Yagmur B. (2007). The effects of calcium sulphate on growth, membrane stability and nutrient uptake of tomato plants grown under salt stress. . Environ. Exp. Bot. 59: 173-178.

Turhan E. and Eris A. (2006). Growth and stomatal behaviour of two strawberry cultivars under long-term salinity stress. Turk. J. Agric. For. 31: 55-61

Volkmar K. M., Hy Y. and Steppuhn H., (1998). Physiological responses of plants to salinity: A review. Can. J. Plant Sci. 78: 19-27.

$$
\begin{aligned}
& \text { استجابه ثلاثة أصناف شائعه الاتتشار من الفراولة للملوحة في الأردن } \\
& \text { سائد جوزيف عويس } \\
& \text { قسم وقاية النبات و الإدارة المتكاملة للآفاتـ كلية الزر اعة الأرد } \\
& \text { جامعة مؤتة ـ الكرك- الاردن ل الآن }
\end{aligned}
$$

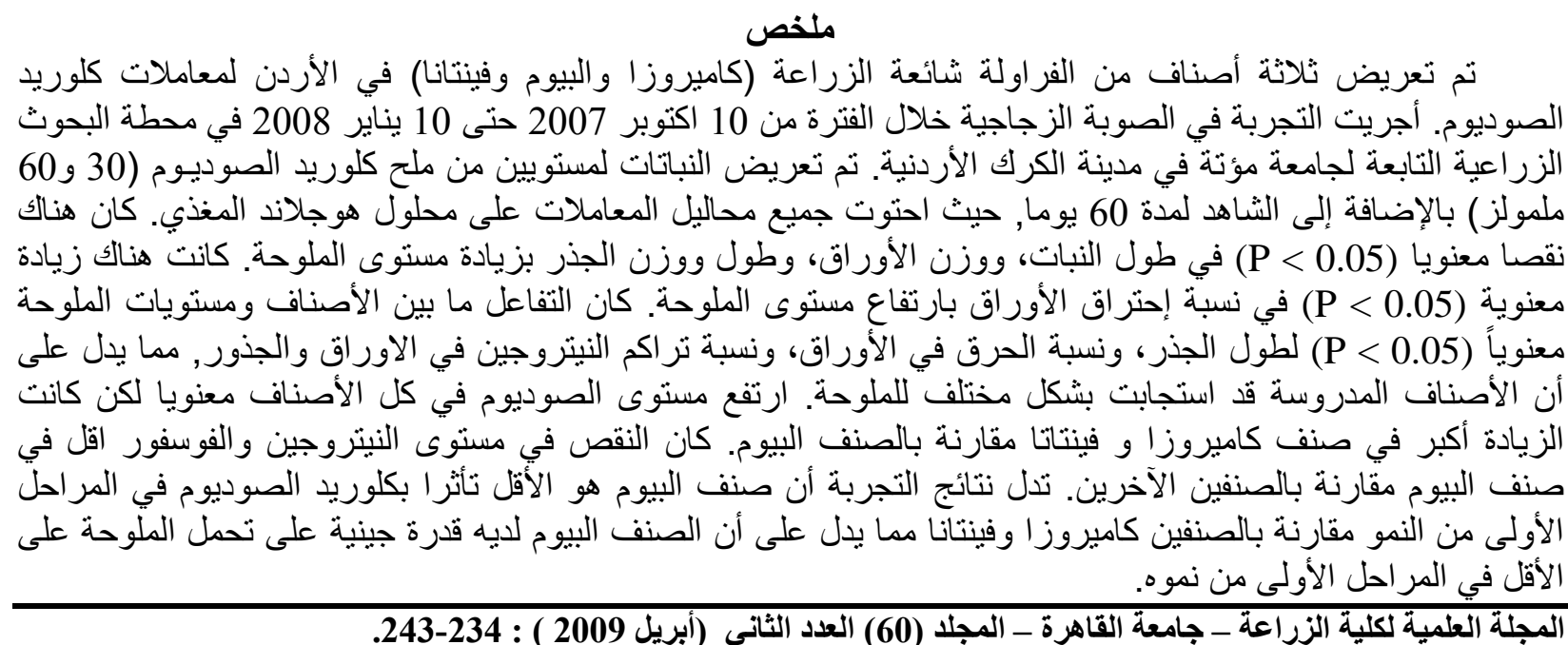

